University of Windsor

Scholarship at UWindsor

Spring 2015

\title{
Racial Identity Profiles of Asian-White Biracial Young Adults: Testing a Theoretical Model With Cultural and Psychological Correlates
}

Vanessa Chong

B.C.H Kuo

University of Windsor

Follow this and additional works at: https://scholar.uwindsor.ca/psychologypub

Part of the Psychology Commons

\section{Recommended Citation}

Chong, Vanessa and Kuo, B.C.H. (2015). Racial Identity Profiles of Asian-White Biracial Young Adults: Testing a Theoretical Model With Cultural and Psychological Correlates. Asian American Journal of Psychology.

https://scholar.uwindsor.ca/psychologypub/38

This Article is brought to you for free and open access by the Department of Psychology at Scholarship at UWindsor. It has been accepted for inclusion in Psychology Publications by an authorized administrator of Scholarship at UWindsor. For more information, please contact scholarship@uwindsor.ca. 


\begin{abstract}
While the biracial population is expected to grow at astonishing rates in the upcoming decades across North America, rigorous quantitative psychological research on biracial identity is currently scarce. Therefore, the purpose of the present study was to examine biracial identity profiles in a large sample of Asian-White biracial young adults $(N=330$; aged $18-30)$ living in the U.S. and Canada, as well as assess the interrelationships among biracial identity and psychological adjustment variables. Grounded in the expanded theoretical model of Multiracial Heritage Awareness and Personal Affiliation (M-HAPA: Choi-Misailidis, 2004) and its corresponding biracial identity measure, cluster analysis was conducted to evaluate participants' 'patterns' or 'profiles' of scores on biracial identity orientation subscales. Three unique biracial identity groups emerged: the Asian-White Integrated, the Asian Dominant, and the White Dominant groups. Between-group differences on participants' measures of cultural socialization, psychological distress and internalized oppression were analyzed and compared. The AsianWhite Integrated group reported more cultural socialization than the other two groups. Furthermore, Asian Dominant participants showed the highest levels of psychological distress, whereas White Dominant participants showed the highest levels of internalized oppression among all groups. The results lend empirical support to the study's hypotheses and the M-HAPA model. Theoretical, conceptual, and methodological implications for future biracial identity research are discussed.
\end{abstract}

Keywords: biracial identity, cultural socialization, psychological adjustment, internalized oppression

First published in Asian American Journal of Psychology 10.1037/aap0000022. Copyright American Psychological Association 2015. 
Racial identity is an essential component of self-meaning, especially among racial minorities. It encompasses the ways persons of color understand themselves in relation to others and the larger society. As such, racial identity can significantly impact individuals' self-concept, well-being, and relationships. Against the backdrop of racial stratification, discrimination, and racism in North America, the racial identity development process for ethnic minorities can be complicated. This is especially true for biracial individuals, who straddle age-old racial divides and challenge the very meaning of race.

The biracial population in North America is growing at astounding rates. According to Root (1996), the number of multiracial births in the United States has increased by $260 \%$ since the 1970 s, as compared to $15 \%$ for the number of monoracial births. In $2012,2.9 \%$ of the population in the United States (9.1 million people) identified themselves as having a mixed-race background (U.S. Census Bureau, 2012). It is estimated that by the year 2050, one in five people will identify with more than one race in the U.S. (Farley, 2001). Similarly, 2.7\% of Canada's total population reported a mixture of European heritage and at least one non-European heritage in 2006 (Statistics Canada, 2006). This number represented an increase of $25 \%$ since the previous census in 2001. The Asian-White mixed race population is growing at particularly fast rates. It was reported that between 2000 and 2010, the Asian-White population in the U.S. increased by $87 \%$ to 1.6 million individuals, the highest total in history (Jones \& Bullock, 2012). However, presently research on Asian-White biracial identity is very scarce (Shih \& Sanchez, 2005).

\section{Theoretical and Methodological Issues in Biracial Identity Research}

Our understanding of the biracial identity experience has changed a great deal over the past 80 years (Thornton, 1996). Earlier theories that took the Problem Approach assumed that biracial 
individuals were marginalized and therefore tended to have more psychological problems than monoracial individuals (e.g., Stonequist, 1937). Starting in the 1970s, theorists began to take the Equivalent Approach, assuming that healthy biracial individuals undergo racial identity development processes similar to those of monoracial minorities (e.g., Porterfield, 1978). In the 1990s, in response to the growing recognition of the uniqueness of biracial identity, models of biracial identity began to take the Variant Approach (e.g., Kerwin \& Ponterotto, 1995; Poston, 1990). According to this approach, biracial identity development is qualitatively distinct from monoracial identity, but it failed to account for identity fluidity. More recent biracial identity models have taken an Ecological Approach (Rockquemore, Brunsma, \& Delgado, 2009). These models focus on the range of identity orientations available for biracial individuals to choose from and the fluid nature of biracial identity (Choi-Misailidis, 2004; Root, 1997). They represent the most contemporary view on the biracial identity experience.

There have been some methodological challenges with measuring biracial identity. In the past, biracial identity models have been developed based on case studies or qualitative studies with a small number of participants (e.g., Gillem, Cohn, \& Thorne, 2001). More recently, however, researchers have strived to measure identity fluidity in terms of ecological models. For example, Rockquemore and Brunsma (2002) proposed a "taxonomy of racial identity options", which include a Singular Identity (i.e., identifying as exclusively Black or White), a Border Identity (i.e. identifying as exclusively biracial), a Protean Identity (i.e., fluidly switching between identities), and a Transcendent Identity (i.e., not identifying with race at all). To measure this model, biracial respondents are categorized into groups based on their answer to a single item. This method, however, failed to assess the degree to which biracial individuals relate to each identity type and assumed each identity type was a separate, discrete variable. 
While Rockquemore and Brunsma's taxonomy attempts to take into account identity fluidity in the form of Protean Identity, in reality this identity group may still be quite heterogenous. For example, while some Asian-White biracial individuals categorized in the Protean Identity group may identify with their Asian heritage more often or in more situations, while others in the same group may identify with their White heritage more often or in more situations. Hence, this conceptual taxonomy is unable to make a nuanced distinction among biracial individuals.

Considering the foregoing theoretical and measurement issues pertaining to biracial identity, the Multiracial Heritage and Awareness Personal Affiliation (M-HAPA) model by Choi-Misailidis (2004) is a new ecological model that attempts to address some of these concerns. The M-HAPA model uses a multidimensional framework (as opposed to a categorical one) and employs the Multiracial Heritage and Personal Affiliation Scale (M-HAPAS) to assess biracial identity. The M-HAPA model accounts for identity fluidity by describing three biracial identity orientations: 1) Singular Identity (i.e., identifying with only one heritage group); 2) Integrated Identity (i.e., identifying with multiple heritage groups); and 3) Marginal Identity (i.e., not identifying with either heritage group and having a sense of alienation from both heritage groups). Additionally, the M-HAPA model proposes the notion of identity 'dominance' to account for the fact that biracial individuals often have a primary biracial identity orientation with which they identify more strongly and more often. Thus, this model offers a middle ground in addressing the issue of context-based identity fluidity versus trait-like identity dominance.

In terms of improving upon existing biracial identity measurement issues, the M-HAPAS assesses each respondent's score on each identity subscale based on his/her answers to multiple items on the questionnaire. To the authors' knowledge, this scale represents the only available multidimensional multiracial identity measure that was theoretically derived and empirically 
tested with a large sample ( $N=364$ multiracial adults aged 17-58 from three universities in Hawaii). For these reasons, the M-HAPA model and its corresponding measure were adopted in the present investigation.

\section{Psychological and Contextual Influences on Asian-White Biracial Identity}

Based on the authors' review of the literature, no published quantitative studies have specifically investigated psychological adjustment among Asian-White biracial individuals at the time of this research. Existing studies have suggested that those who identify primarily with their majority heritage group tend to have poorer psychological adjustment than those who identify with their minority heritage group or both heritage groups (Binning, Unzueta, Huo, \& Molina, 2009; Lusk, Taylor, Nanney, \& Austin, 2010). These studies have investigated biracial samples with a wide range of heritage backgrounds, including Asian, White, African American, Hispanic, and Native American. Furthermore, findings from Black-White biracial identity research suggest that those individuals who do not identify with either of their heritage groups are the most psychologically vulnerable (Coleman \& Carter, 2007; Lusk et al., 2010).

At the present time, the generalizability of these findings to the Asian-White biracial population is unclear. Research has suggested that biracial subgroups may differ in terms of their racial identity experiences. For example, Lou, Lalonde, and Wilson (2011) found that AsianWhite participants were more likely than their Black-White counterparts to identify with Rockquemore and Brunsma's (2002) Protean Identity. Similarly, Harris and Sim (2002) found that, as compared to Black-White participants, Asian-White participants had less consistent racial identities between the home and school settings. This identity fluidity may be related to the importance placed on interdependence and maintaining harmony among Asian cultures. In addition, Asian immigrants have been considered a "model minority" group and tend to be 
perceived more positively than other minority groups (e.g., African Americans) in North America (Berry, 2006; Sue \& Sue, 2003). Thus, it can be argued that the Asian-White biracial identity experience is impacted by unique sociopolitical and cultural forces in North America that may set it apart from the experiences of other biracial groups (e.g., Black-White).

Contextual factors are clearly important in considering biracial identity. A prominent biracial identity researcher in the field, Maria Root, asserted that contextual factors can be likened to lenses, influencing the ways in which different situations and experiences are perceived by biracial individuals (Root, 1998). Root identified several important "macrolenses", including gender, class, and the regional history of race relations, as well as several "microlenses", including inherited factors (e.g., cultural values), traits (e.g., coping skills), and social environments (e.g., the home). This is consistent with observations from the monoracial identity literature, suggesting that family cultural socialization (e.g., being taught the beliefs, values, and traditions of their cultures) can have a profound effect on racial identity (Gartner, Kiang, \& Supple, 2013; Motomura, 2007).

Internalized oppression constitutes yet another critical factor that impacts biracial individuals' racial identification process (Rockquemore \& Laszloffy, 2005). It often involves the individual holding "Whiteness" in high regard while feeling ashamed of his/her minority heritage. Pyke (2010) noted that ethnic minorities often experience "intraethnic othering." This occurs when they denigrate fellow members of their ethnic group who are perceived to be "too ethnic." As such, internalized oppression reflects both microlense and macrolense factors, in that a biracial person's social environments and broader historical and societal forces simultaneously contribute to the shaping of his/her self-perception. While internalized oppression is a critical 
variable in understanding monoracial and biracial minorities, it has been largely ignored in the biracial identity and the larger cross-cultural psychology literature (Pyke, 2010).

Our current understanding of internalized oppression is based largely on research with monoracial African Americans. Internalized oppression has been linked to a wide variety of psychological problems among racial minorities, including perceived stress, anxiety, and depression (Tull, Sheu, Butler, \& Cornelious, 2005; Tull et al., 1999). With respect to research on Asian internalized oppression, David and colleagues have coined the term "colonial mentality", referring to a form of internalized oppression experienced among Filipinos and Filipino Americans (David, 2008). Individuals who scored higher on colonial mentality reported less secure ethnic identities, lower self-esteem, and more depression (David, 2008; David \& Nadal, 2013; David \& Okazaki, 2006).

The notion of internalized oppression has been incorporated into a few biracial identity models (e.g.

, Poston, 1990; Rockquemore \& Laszloffy, 2005; Root, 1990). For example, Poston (1990) described the "Enmeshment/Denial" stage involving self-hatred and embarrassment about one parent, who is usually the minority parent. Virtually no studies have been conducted on internalized oppression among biracial individuals. However, in a study conducted by Harrison (1997), 60\% of the Black-White female sample admitted to having lied in the past about their racial backgrounds. Those who identified themselves as being "biracial but predominantly White" were more likely to report that they sometimes felt conflicted because they wished they were part of a White family. Strikingly, $26 \%$ percent of the sample reported that if they could be 
born again, they would want to be monoracial. Despite growing evidence, to date no studies have directly and quantitatively investigated internalized oppression among biracial individuals.

\section{The Current Study}

At the conceptual level, the current study examined the relationship between biracial identity and psychological adjustment in Asian-White biracial individuals, a subgroup that has been largely overlooked in the literature. Additionally, the current study examined the effects of internalized oppression, a critically understudied variable for racial minorities. Given the importance of contextual variables in shaping biracial identity (Root's 1998), the present study adopted a contextual perspective by further examining the constructs of cultural socialization and internalized oppression. In short, the current study attempted to address some of the critical gaps in the biracial literature. To this end, the present research: a) focused on the study of the AsianWhite biracial population and its identity subgroups; b) employed quantitative measures to assess biracial identity and its psychological and contextual correlates, and c) recruited a large sample of Asian-White biracial participants in U.S. and Canada.

At the methodological level, the current study strived to improve on the measurement of biracial identity by adopting a multidimensional scale. Going beyond the single-item assessment and categorization approach, this study utilized a modified version of the multidimensional MHAPAS measure (Choi-Misailidis, 2004) to assess Asian-White biracial participants' identity profiles based on patterns of identity orientation scores. This approach enabled the researchers to not only discern identity groupings among biracial participants more precisely, but also to reveal identity fluidity (i.e., higher scores on multiple identity subscales) and dominance (i.e., one identity subscale score being higher than others for a given identity profile) among biracial respondents. This represents a novel and unique methodological undertaking. 
The present study asks three research questions. First, "Which type of biracial identity pattern is most closely associated with psychological distress among Asian-White young adults?" On the basis of previous research, it was hypothesized that individuals who primarily identify with both their Asian and White heritages would have less psychological distress than those who primarily identify with one of their heritages (Hypothesis 1). Second, "To what extent is cultural socialization associated with biracial identity development among Asian-White young adults?" It was expected that greater cultural socialization (to both one's Asian and White heritages) would be correlated with a more integrated biracial identity (Hypothesis 2). Finally, "What are the interrelationships between internalized oppression, biracial identity, cultural socialization, and psychological distress among Asian-White young adults?" It was hypothesized that more internalized oppression would be associated with greater identification with one's White heritage, less cultural socialization, and more psychological distress (Hypothesis 3).

\section{Method}

\section{Participants and Procedures}

Asian-White biracial participants between the ages of 18 and 30 were recruited from both the United States and Canada, in order to maximize sample size and statistical power. Even though differences in interracial group relations have been noted between U.S. and Canada, previous biracial studies have combined American and Canadian biracial samples and did not find significant differences on racial identity and variables (e.g., Lou et al., 2011). Furthermore, in the present study no significant differences on the key variables were found between biracial participants recruited from the U.S. vs. Canada. Thus, participants from both countries were combined in the analyses. 
To be eligible, participants had to have one White parent and one parent of East Asian descent. This group was selected because of the commonalities in cultural values as noted in the literature, including interdependence, loyalty, obligation, respect for authority, and maintaining interpersonal harmony (Uba, 1994). A multi-pronged recruitment method was used which included recruitment through: 1) the administrators of Facebook interest groups related to biracial identity ( $78 \%$ of the total sample); 2) the "snowball technique," wherein the first author's friends and family members were asked to forward the recruitment e-mail to their own personal contacts $(14 \%) ; 3)$ the directors of various ethnic-specific community groups and organizations $(3 \%) ; 4)$ undergraduate students through the University of Windsor's Psychology Participant Pool (1\%), and 5) e-mailing administrative staff for students in other academic departments across the University of Windsor $(0.1 \%)$. A web survey was used. As an incentive for participation, those who completed the study were entered in a draw for one of six $\$ 25$ gift certificates for a popular online shopping website.

The final sample was comprised of 330 Asian-White biracial young adults $(76 \%$ female, $23 \%$ male, $1 \%$ other gender) with the mean age of $23(\mathrm{SD}=3.8)$. In total $73 \%$ of the participants reported living in the U.S. while $24 \%$ reported living in Canada. Sixty-six percent were born in the U.S., $20 \%$ were born in Canada, and 14\% were born outside of North America. The majority of the participants $(74 \%)$ reported having fathers who were White and mothers who were Asian.

\section{Measures}

Biracial identity. Biracial identity was measured with an adapted version of the Multiracial Heritage Awareness and Personal Affiliation Scale (M-HAPAS: Choi-Misailidis, 2004) - a measure developed with a sample of 364 biracial and multiracial students in Hawaii with Asian, Black, Hispanic, Caucasian, Pacific Islander, and Native American backgrounds. 
Fifty-two percent of the sample identified Asian as part of their heritage. In the original 43-item M-HAPAS, respondents are asked to rate the degree to which they agreed with each item on a Likert scale, from 1 (strongly disagree) to 7 (strongly agree). Choi-Misailidis' original theory proposed that there were three identity orientations: integrated identity, singular identity, and marginal identity. However, a factor analysis of the original scale yielded four subscales: integrated-combinatory (identifying with multiple heritages), integrated-universality (identifying with people of all races), singular (identifying with one heritage), and marginal (not identifying with any heritage). Respondents received a score on each subscale, with higher scores indicating stronger attitudes related to that identity status.

In the original instrument development study, Choi-Misailidis (2004) measured singular identity status as a single identity orientation, without accounting for the fact that identifying with one's majority heritage (i.e., White European) can be qualitatively different from identifying with one's minority heritage (i.e., Asian). Consequently, the original M-HAPAS singular subscale was made into two subscales, one assessing participants' orientation toward their minority group (singular-minority identity) and the other assessing their orientation to the majority group (singular-majority identity). As such, slight wording changes were made to the two modified singular identity subscales. Specifically, references to one's mother's heritage/group, father's heritage/group, and parent heritage/group were removed and were replaced with references to one's "White heritage" and "minority heritage". The wording of some items was modified in order to make items more specific to the current study. For instance, the item "Others remind me frequently that I am different" was changed to "Others remind me frequently that I am racially different". These modifications were based on feedback from a focus group of three doctoral students who were familiar with multicultural psychology research. 
The version of the M-HAPAS used for this study consisted of 56 items with five hypothesized subscales: integrated-combinatory, integrated-universality, singular-minority, singular-majority, marginal.

Since the M-HAPAS has not been validated with additional samples beyond the sample used in Cho-Misailidis' original study (2004) and the original items were modified for the current study, a confirmatory factor analysis (CFA) of the scale was conducted. Structural equation modelling (SEM) was used to assess the psychometrics of the modified M-HAPAS. Based guidelines suggested by Byrne (2010) and Lei and Lomax (1999) ${ }^{1}$, criteria were not met for the hypothesized five-factor solution. As a follow-up, an exploratory factor analysis (EFA) was conducted, using the Common Factor Analysis with the principle axis factoring technique and direct oblimin rotation. Visual inspection of a scree plot suggested a four-factor solution. Together, the four factors accounted for $49.02 \%$ of the variance. Items with factor loadings greater than or equal to .40 in the pattern matrix were retained (Stevens, 2002). This resulted in the retention of 46 items for the modified M-HAPAS, with factor loadings ranging from .40-.88.

The retained items were inspected and interpreted for each factor. Factor 1 (eigenvalue 9.43) was named Integrated Identity (identifying with one's Asian and White heritages with equal importance). This factor consisted of 13 items and accounted for $19.24 \%$ of the total variance. Factor loadings ranged from -.40 to -.83. Factor 2 (eigenvalue 7.26 ) was named Singular-Majority Identity (identifying with one's White heritage). This factor consisted of 11 items and accounted for $14.82 \%$ of the total variance. Factor loadings ranged from .42 to .86 . Factor 3 (eigenvalue 4.55) was named Marginal Identity (being alienated from both Asian and White heritages). This factor consisted of 10 items and accounted for $9.29 \%$ of the total variance.

\footnotetext{
${ }^{1}$ These guidelines suggest that acceptable model fit requires Chi Square $\mathrm{p}$ values exceeding .05, RMSEA values below .08, and CFI values = exceeding .90 .
} 
Finally, Factor 4 was named Singular-Minority Identity (identification with one's Asian heritage). This factor consisted of 12 items and accounted for $5.67 \%$ of the total variance.

The final version of the M-HAPAS used in this study was comprised of 46 items and four subscales: Integrated Identity (13 items), Singular-Majority Identity (11 items), Marginal Identity (10 items), and Singular-Minority Identity (12 items). Internal consistencies for each of these subscales were high $(\alpha=.90, .90, .86$, and .90 , respectively). The integrated-combinatory and the integrated-universality subscales, which Choi-Misailidis supplemented to account for an unexpected four-factor solution in the original M-HAPAS development study, were not supported in the current analysis. However, the original theoretical M-HAPA model was supported.

Cultural socialization. The Family Ethnic Socialization Measure (FESM; Umaña-Taylor \& Fine, 2001) is a 12-item measure of one's perceptions of family cultural socialization attitudes, beliefs, and practices. Respondents are asked to rate their level of agreement with items on a Likert scale, from 1 (not at all) to 5 (very much). The item scores are summed, with higher total scores indicating a higher degree of perceived cultural socialization in one's family-of-origin. The FESM has demonstrated internal consistency in an ethnically diverse sample of 615 college students (Study 1) and 231 high school students (Study 2) ( $\alpha=.92$ to .94) (Umaña-Taylor, Yazedjian, \& Bámaca-Gómez, 2004). The measure has also been shown to have construct validity (Umaña-Taylor et al., 2004).

The FESM was designed for use with monoracial youths. For the purpose of the current study, items were changed from present tense to past tense, as the young adult participants in the current study were asked to rate their family cultural socialization retrospectively during their younger years. Additional instructions were added to the beginning of the survey, directing 
participants to reflect on their cultural socialization experiences during childhood ${ }^{2}$. Each participant was presented with two versions of the FESM. In the first version, participants were asked to rate their cultural socialization with respect to their Asian culture. In the second version, participants were asked to rate their cultural socialization with respect to their White/European culture. Internal consistencies were good for both the Asian and White/European versions in the current study ( $\alpha=.93$ and .89 , respectively). Item-total correlations ranged from .41 to .85 for the Asian subscale and from .29 to .76 for the White subscale.

Psychological distress. The Brief Symptom Inventory-18 (BSI-18; Derogatis, 2000) is a self-report screening tool for psychological distresses and disorders. Respondents are asked to rate the degree to which they have experienced various symptoms in the past week on a fivepoint scale, from 0 (not at all) to 4 (extremely). A Global Severity Index (GSI) represents an overall score of psychological distress. This measure has been used with a previous sample of Asian international students and showed good internal consistency (alpha=.88) (Wang \& Mallinckrodt, 2006). The original Brief Symptom Inventory, of which the BSI-18 is a shortened version, also showed good internal consistency in a sample of multiracial participants (alpha=.96) (Sparrold, 2003). In the present study, the Cronbach's alpha was .91 for the GSI, demonstrating support for the internal consistency of the BSI-18 in the current sample.

Internalized oppression. The internalized oppression measure used in the current study consisted of 34 items; 25 items were adapted from the Colonial Mentality Scale for Filipino Americans (CMSFA) (David \& Okazaki, 2006) and nine items were written by the first author. The scale was named the Internalized Oppression Scale for Biracial Individuals (IOSBI). Respondents were asked to rate these items on a scale from 1 (strongly disagree) to 6 (strongly

\footnotetext{
2 These changes were made with the permission of the test author.
} 
agree). The terms "minority group" and "minority heritage" were defined under the measure instructions. The scores were summed across all the items with higher total scores indicating greater levels of internalized oppression.

Items adapted from the CMSFA were modified to apply to the current study's biracial sample. Some of the CMSFA items that were specifically worded in terms of Filipino facial features, language, and culture were modified to apply more broadly to Asian characteristics (e.g., " In general, I am ashamed of members of my minority group because of the way they dress and act"). Items on the CMSFA that were more specific to the migration history of the Filipino people were omitted. Nine items were also developed by the researchers to reflect some of the experiences unique to biracial individuals based on previous biracial identity research (Brunsma \& Rockquemore, 2001; Motomura, 2007; Root, 1997), theoretical writings (Poston, 1990; Root, 1990), case studies (Rockquemore \& Laszloffy, 2005), and the first author's personal experiences as a biracial individual. For example, four items regarding physical appearance were developed based on research suggesting that physical appearance is particularly salient to biracial individuals' racial identity development (Brunsma \& Rockquemore, 2001) (e.g., "I wish I looked more like my White parent"). Additionally, two items were based on the premise that minority-majority biracial individuals are sometimes ashamed of their parents because of their races, particularly their non-White parents (Poston, 1990; Root, 1990) (e.g., "Sometimes I am ashamed to be seen with my non-White parent"). Three items were added that assess the possibility that some biracial individuals may reject the non-White parts of themselves (Rockquemore \& Laszloffy, 2005). For example, "Sometimes I feel grateful that I am not a fullblooded member of my minority group" was added. The face validity of these items and their wording were verified by the focus group recruited by the first author prior to data collection. 
Due to the fact that most of the CMSFA items were modified and nine new items were added to comprise the IOSBI, an exploratory factor analysis (EFA) was conducted using the principle axis factoring extraction technique with direct oblimin rotation. A scree plot indicated a single factor solution. Based on Stevens' (2002) recommendations, only items with factor loadings greater than .40 were retained. Only one item was removed based on this criterion. The remaining 32 items comprised a single factor and accounted for $41.36 \%$ of the variance (eigenvalue 13.23). Factor loadings of the items ranged from .44 to .82. The internal consistency of the scale was very good $(\alpha=.95)$, and item-total correlations ranged from .40 to .81 . Thus, the IOSBI was judged to be measuring a single factor, internalized oppression.

\section{Cluster Analysis}

Given the research questions and hypotheses (hypothesis 1 and 2) of the present study which involve discerning how different types of biracial identity relate to cultural socialization and psychological variables in Asian White young adults, cluster analysis was used. A two-step cluster analysis using the SPSS computer program was conducted. Each of the four biracial identity orientation variables was entered into this analysis using log-likelihood distances and the Bayesian Information Criterion (BIC). Due to the fact that the order in which cases are entered in a dataset can result in different outcomes, randomized case order for cluster analysis was necessary and applied in this case (Nourisis, 2010). The final solution resulted in three clusters. A one-way between subjects analysis of variance(ANOVA) was conducted for each biracial identity orientation (integrated, singular-majority, singular-minority, marginal) for each cluster group (cluster 1, cluster 2, cluster 3). Subsequently, post-hoc Games-Howell tests were conducted to aid in the cluster interpretation. 
The researchers were also interested in investigating the between-cluster differences in cultural socialization variables (Asian and White cultural socialization), and psychological adjustment variables (psychological distress, internalized oppression). Hence, a subsequent series of four separate ANOVAs was conducted. A Bonferroni corrected significance criterion of $p=.0125$ corrected for inflation of Type I error.

\section{Results}

\section{Overall Cluster Profile and Identification}

Judging by scores based on the Modified M-HAPAS, individuals in Cluster $1(n=132)$ were characterized by higher singular-minority identity scores than the other two clusters ( $p s<.001)$ (See Table 1). Moreover, those in Cluster 1 had higher singular-majority identity scores than those in Cluster 2 but lower singular-majority identity scores than those in Cluster 3 ( $p s<.001)$. They also reported lower integrated identity scores than those in Cluster $2(p<.001)$ and higher marginal identity scores than those in the other two clusters $(p<.001)$. However, those in Cluster 1 did not differ significantly on the integrated identity scores from those in Cluster 3. Based on this profile, this group of participants was labelled Asian Dominant.

On the other hand, individuals in Cluster $2(n=132)$ tended to report higher integrated identity scores than those in Clusters 1 and 3 ( $p s<.001)$. Additionally, they scored lower on singular-majority than those in Clusters 1 and 3 ( $p s<.001)$. Marginal identity scores were lower among those in Cluster 2 than in Clusters 1 and 3 ( $p<.001$ and $p<.006$, respectively). Singularminority identity scores were lower among Cluster 2 individuals, as compared to those in Cluster $1(p<.001)$, but were not significantly different from those in Cluster 3. Given the seemingly 'balanced' nature of their identity pattern, this cluster was labelled Asian-White Integrated. 
Cluster 3 ( $n=63$ ) was characterized by higher singular-majority identity scores than those in Clusters 1 and 2 and lower integrated identity scores than those in Cluster $2(p s<.001)$. Individuals grouped into this cluster also had singular-minority identity scores similar to those grouped into Cluster $2(p=.70)$, but were lower than those in Cluster $1(p<.001)$. Additionally, they tended to have marginal identity scores that were lower than those in Cluster $1(p<.001)$ and higher but those in Cluster 2 ( $p<.008)$. This cluster was labelled White Dominant. Figure 1 provides a visual comparison of biracial identity orientation by the three clusters.

$<$ Insert Table $1 \&$ Figure 1 About Here>

\section{Between-Cluster Differences}

Cultural socialization. The clusters were compared on cultural socialization. For Asian cultural socialization, those in the Asian-White Integrated cluster had higher scores than those in the White Dominant cluster $(p<.001)$. Individuals in the Asian-White Integrated group also scored higher on Asian cultural socialization than those in the Asian Dominant group, but this difference only approached statistical significance $(p=.034)$. For White cultural socialization, those in the Asian Dominant cluster had significantly lower White cultural socialization scores than those in the Asian-White Integrated cluster $(p<.001)$. Those in the Asian Dominant cluster also reported significantly lower White cultural socialization scores than those in the White Dominant cluster $(p=.008)$ and lower White cultural socialization scores than those in the AsianWhite Integrated cluster. However, this comparison only approached statistical significance $(p=.016)$ (Figure 2).

\section{< Insert Figure 2 About Here>}

Psychological adjustment variables. Between-cluster differences on psychological adjustment were also found (Table 1). Games-Howell tests indicated that individuals in the 
Asian-White Integrated cluster were significantly less distressed than those in the Asian Dominant cluster $(p<.001)$ (Figure 2). Those in the White Dominant cluster also reported lower psychological distress scores than those in the Asian Dominant group, although the difference only approached significance $(p=.031)$. All between-cluster comparisons for internalized oppression were significant $(p s<.001)$ (Figure 2). White Dominant individuals had the highest internalized oppression scores, followed by Asian Dominant individuals. Asian-White Integrated individuals had the lowest internalized oppression scores.

\section{Discussion}

The current study is a unique quantitative study of Asian-White biracial identity, which was grounded in an empirically tested model of biracial identity and involved a large sample of Asian-White young adults. The overarching goal of the present investigation was to better understand racial identity and its relationship with cultural socialization and psychological experiences among Asian-White biracial individuals in North America.

Importantly, the current study took a novel approach by categorizing biracial participants based on overall "patterns" or "profiles" of racial identification. This is a clear methodological departure from the conventional yet disputable method of assigning biracial individuals to a racial identity orientation based on his/her answer to a single item. By examining patterns scores on racial identity subscales, it allowed for a more accurate delineation of Asian-White subgroups. Using cluster analysis, three biracial identity groups emerged. Each identity group had its own distinct profile based on differential patterns of scores on the integrated, the singularminority, the singular-majority, and the marginal identity subscales of the M-HAPAS. These three groups were labelled the White Dominant, the Asian Dominant, and the Asian-White 
Integrated groups. Significant between-group profile differences were found on cultural socialization, psychological distress, and internalized oppression.

The results showed that participants who were in the Asian-White Integrated group identified more strongly with both of their parents' heritages in a blended, integrated manner, as opposed to identifying exclusively with either their Asian or White heritages. Their low scores on the marginal identity scale also suggested an overall sense of belonging and social connectedness. Those in the White Dominant group were more likely to identify with their White parent's heritage than those in the other two groups. Paradoxically, they also tended to feel more alienated from both of their heritages than those in the Asian-White Integrated group, but less alienated than those in the Asian Dominant group. Those in the Asian Dominant group identified more strongly with their Asian parent's heritage than those in the other two groups. However, surprisingly they also reported higher singular-majority identity scores than those in the AsianWhite Integrated group. They were also higher than the other two groups on marginal identity, a measure of the extent to which they feel disconnected from both of their heritages.

When between-group differences on psychological distress were examined, individuals in the Asian-White Integrated group reported lower psychological distress than those in the White Dominant group. As such, Hypothesis 1 was supported. This finding is consistent with previous research which has demonstrated that biracial individuals who are more connected with both of their heritages tend to experience better psychological health (Choi-Misailidis, 2004; Lusk et al., 2010). This may be explained by social identity theory (Tajfel \& Turner, 1986). That is, AsianWhite biracial individuals who identify with both heritages may have a greater sense of attachment to and belonging in multiple groups. This may in turn result in more positive selfconcept and psychological adjustment. In addition, feeling connected to multiple cultures may 
provide a wider range of values, lessons, and sources of social support for these individuals to draw on when faced with life stressors (Shih \& Sanchez, 2005).

The fact that those in the Asian Dominant group had higher singular-majority scores than those in the Asian-White Integrated group seems to be counterintuitive. However, these results may give us a glimpse into the between-group differences in the biracial identity process. It is possible that those in the Asian Dominant group may have regarded the boundaries between their component heritages (i.e., Asian and White) in a more clear-cut manner than those in the AsianWhite Integrated group. By contrast, those in the Asian-White Integrated group may believe that they can combine, balance, and integrate their component heritages (Rockquemore \& Laszloffy, 2005). This "one-or-the-other" perspective of the Asian Dominant group may have resulted in higher singular-majority scores than the blended style of Asian-White Integrated group members.

The current study also suggests that cultural socialization may play an important role in influencing biracial identity development. As predicted under Hypothesis 2, those in the AsianWhite Integrated group reported more Asian cultural socialization than those in the White Dominant and the Asian Dominant groups. Those in the Asian Dominant group reported having less exposure to White cultural socialization than those in the White Dominant and the AsianWhite Integrated groups. These results are consistent with previous research suggesting that cultural socialization has a direct impact on racial identity (Gartner et al., 2013).

Another valuable contribution of the present study was the examination of internalized oppression using quantitative methods with a biracial sample. In support of Hypothesis 3 , the present study found that those in the White Dominant identity group were more likely to value their majority heritage and denigrate their minority heritage. It is possible that Asian-White biracial individuals who identify more with the White Dominant group may be engaging in an 
active rejection of their Asian heritage. Incidentally, these results parallel findings from previous studies of monoracial African Americans, in which those in the pre-encounter stage of racial identity development (i.e., less identification with their African American heritage) reported higher levels of internalized oppression (Cokley, 2002). The present findings further align with Root's (1990) prediction that biracial individuals who experience internalized oppression may over-identify with one (usually White) heritage and may attempt to gain approval from this "hierarchically superior group."

Unexpectedly, the present study found that individuals in the Asian Dominant group reported significantly higher levels of internalized oppression than those in the Asian-White Integrated group. This finding was somewhat surprising because internalized oppression often involves biracial individuals' rejection of their minority (in this case, Asian) heritage. The authors expected that those who identified primarily with their Asian Dominant group would have low levels of internalized oppression. This finding may provide insight into between-group discrepancies in the identity consolidation process among mixed-race individuals. For instance, it is likely that members of the Asian-White Integrated group may see their component identities as compatible, and as a result may feel less ambivalent about being biracial. This could lead them to feel more secure about their identities, resulting in less internalized oppression. By contrast, those in the Asian Dominant Group may have greater identity ambivalence. As a result, they may frequently flip between different modes of racial identification. Indeed, in a study of mixed race individuals by Cheng and Lee (2009), the researchers found that greater perceived "racial distance" (i.e., beliefs that racial heritages are separate) and "racial conflict" (i.e., feeling tension between racial heritages) were associated with less multiracial pride. 
Alternatively, the surprising finding that Asian Dominant individuals reported more internalized oppression than Asian-White Dominant individuals may reflect the need for a more fine-tuned examination of internalized oppression. In the original validation study for the Colonial Mentality Scale for Filipino Americans, on which the current study's internalized oppression scale was based, David and Okazaki (2006) identified both covert manifestations of colonial mentality (e.g., shame about being Filipino) and overt manifestations of colonial mentality (e.g., behaviours to distance oneself from his/her Filipino heritage, such as discriminating against members of their own group). They found that both forms of colonial mentality were negatively correlated with acculturation to the Filipino culture, whereas only overt colonial mentality was positively correlated with acculturation to the American culture. The authors suggested that even though some Filipino Americans may feel ashamed of their Filipino culture, this may not necessarily motivate them to adopt the dominant culture. Similarly, it is possible that Asian Dominant individuals experience shame about their Asian heritage without necessarily feeling connected to their White heritage. That is, White ethnic identity and internalized oppression, or at least covert forms of internalized oppression, may be mutually exclusive. Future research should investigate overt and covert internalized oppression and whether there are significant between-group differences based on biracial ethnic identity.

In the current study, the relative levels of internalized oppression found across the White Dominant, the Asian Dominant, and the Asian-White Integrated groups may also reflect identity stages described in variant approach models of biracial identity (e.g., Poston, 1990). These models propose that biracial individuals first go through a period of heritage rejection and then a period of identity conflict and experimentation, followed by a period of identity integration. It is plausible that higher levels of internalized oppression among the Asian Dominant participants 
reflected a normal developmental phase. Future research should incorporate longitudinal designs, to help track biracial individuals' changes in internalized oppression over time and across different phases of life. Similarly, future research should differentiate between those who experience brief internalized oppression as an expected phase of racial identity development and those who experience chronic internalized oppression in adulthood.

The results of the cluster analysis highlighted three unique racial identity orientations, each of which was characterized by a primary identity orientation. At the same time, participants did show some flexibility in identifying across multiple biracial identity orientations. These findings lend further support to the M-HAPA model's emphasis on both identity dominance and fluidity, which paints a richer, more complex picture of the biracial identity experience. At the same time, it should be noted that the current study focused on biracial identity based on a minority group (Asian)-majority group (White) combination. The extent to which the present findings apply to biracial individuals with a minority group-minority group combination (e.g., Asian-Black) is unclear. Future research should explore and compare the racial identity development processes and experiences of internalized oppression between those with an Asian-White background and those with an Asian-other racial minority heritage background.

The results of the current study should be interpreted with a number of limitations in mind. First, due to the lack of quantitative research and empirically-validated measures available in the prevailing biracial identity literature, the researchers had to adapt measures for cultural socialization and internalized oppression from existing instruments developed for monoracial groups. Although the internal consistencies and the item-total correlations were found to be favorable, the validity of these measures has not been tested with any other biracial samples. Second, prior to the present study, the M-HAPAS had only been tested once in a single 
dissertation study based on a sample of diverse biracial and multiracial individuals in Hawaii (Choi-Misailidis, 2004). Moreover, this scale was modified and expanded for the purpose of the current investigation. Differences in sample composition and these scale modifications might have contributed to the different factor structure for the current study's measure, as compared to that of the original M-HAPAS. While the M-HAPAS's correlations with other key variables were significant in the expected directions, the construct validity of the scale for the current sample cannot be ascertained. Hence, the present study serves as a jumping off point for further research on the M-HAPAS.

\section{Conclusion}

The biracial population in North America is expected to grow at exponential rates in the upcoming decades. Psychological research needs to respond to this rapidly expanding segment of the population by establishing a new understanding of the interactions among individual, family, sociopolitical and contextual factors and how they affect the well-being of biracial individuals. Cast in this light, the current study offers a nuanced examination of the complex psychological and contextual processes associated with biracial identity development among Asian-White young adults living in North America. The current research represents a step forward in advancing the theoretical development and quantitative study of Asian-White biracial identity specifically, as well as biracial identity research more generally. 


\section{References}

Berry, J.W. (2006). Mutual attitudes among immigrants and ethnocultural groups in Canada. International Journal of Intercultural Relations, 30, 719-734. doi:10.1016/j.ijintrel.2006.06.004

Binning, K. R., Unzueta, M. M., Huo, Y. J., \& Molina, L. E. (2009). The interpretation of multiracial status and its relation to social engagement and psychological well-being. Journal of Social Issues, 65(1), 35-49. doi:10.1111/j.1540-4560.2008.01586.x

Brunsma, D. L. \& Rockquemore, K. A. (2001). The new color complex: Appearances and biracial identity. Identity Special Issue: Diasporas and Transnational Identities, 1(3), 225246. doi:10.1207/S1532706XID0103_03

Byrne, B. M (2010). Structural equation modeling with AMOS ( $2^{\text {nd }}$ ed.). New York: Routledge. Cheng, C., \& Lee, F. (2009). Multiracial identity integration: Perceptions of conflict and distance among multiracial individuals. Journal of Social Issues, 65(1), 51-68. doi:10.1111/j.1540-4560.2008.01587.x

Choi-Misailidis, S. (2004). Multiracial-heritage awareness and personal affiliation: Development and validation of a new measure to assess identity in people of mixed race descent. Dissertation Abstracts International: Section B: The Sciences and Engineering, 64 (7-B).

Cokley, K. O. (2002). Testing Cross's revised racial identity model: An examination of the relationship between racial identity and internalized racialism. Journal of Counseling Psychology, 49(4), 476-483. doi:10.1037//0022-0167.49.4.476

Coleman, V. H., \& Carter, M. M. (2007). Biracial self-identification: Impact on trait anxiety, social anxiety, and depression. Identity, 7(2), 103-114. doi:10.1080/15283480701326018 
David, E. J. R. (2008). A colonial mentality model of depression for Filipino Americans. Cultural Diversity and Ethnic Minority Psychology, 14(2), 118-127. doi:10.1037/10999809.14.2.118

David, E.J.R. \& Nadal, K. (2013). The colonial context of Filipino American immigrants' psychological experiences. Cultural Diversity and Ethnic Minority Psychology, 19(3), 298309. doi: $10.1037 / \mathrm{a} 0032903$

David, E. J. R., \& Okazaki, S. (2006). The Colonial Mentality Scale (CMS) for Filipino Americans: Scale construction and psychological implications. Journal of Counseling Psychology, 53(2), 241-252. doi:10.1037/1099-9809.12.1.1

Derogatis, L.R. (2000). BSI-18: Brief Symptom Inventory 18: Administration, scoring, and procedures manual. Minneapolis, MN: National Computer Systems.

Farley, R. (2001). Identifying with multiple races: A social movement that succeeded but failed? (Population Studies Center Report 01-491). Ann Arbor, MI: University of Michigan.

Gartner, M., Kiang, L., \& Supple, A. (2013). Prospective links between ethnic socialization, ethnic and American identity, and well-being among Asian-American adolescents. Journal of Youth and Adolescence, October. doi: 10.1007/s10964-013-0044-0

Gillem, A. R., Cohn, L. R., \& Thorne, C. (2001). Black identity in biracial Black/White people: A comparison of Jacqueline who refuses to be exclusively black and Adolphus who wishes he were. Cultural Diversity and Ethnic Minority Psychology, 7(2), 182-196. doi:10.1037/1099-9809.7.2.182

Harris, D. R., \& Sim, J. J. (2002). Who is multiracial? Assessing the complexity of lived race. American Sociological Review,67(4) 614-627. doi:10.2307/3088948 
Harrison, P. M. (1997). Racial identification and self-concept issues in biracial. Dissertation Abstracts International, 58(04), 2123B.

Jones, N.A. \& Bullock, J. (2012). The two or more races population: 2010. Retrieved from http://www.census.gov/prod/cen2010/briefs/c2010br-01.pdf

Kerwin, C., Ponterotto, J. G., Jackson, B. L., \& Harris, A. (1993). Racial identity in biracial children: A qualitative investigation. Journal of Counseling Psychology, 40(2), 221-231. doi:10.1037/0022-0167.40.2.221

Lei, M., \& Lomax, R. G. (1999). The effect of varying degrees of nonnormality in structural equation modeling. Structural Equation Modeling, 12(1), 1-27.

Lou, E., Lalonde, R. N., \& Wilson, C. (2011). Examining a multidimensional framework of racial identity across different biracial groups. Asian American Journal of Psychology, 2(2), 79-90. doi: 10.1037/a0023658

Lusk, E. M., Taylor, M. J., Nanney, J. T., \& Austin, C. C. (2010). Biracial identity and its relation to self-esteem and depression in mixed Black/White biracial individuals. Journal of Ethnic and Cultural Diversity in Social Work, 19, 109-126. doi:

$10.1080 / 15313201003771783$

Motomura, M. S. (2007). The diverse identities of biracial persons: Antecedent factors, wellbeing and multicultural effectiveness. Dissertation Abstracts International: Section B, $67(7-B), 4160$.

Nourisis, M.J. (2010). 2010 IBM SPSS statistics 19 guide to data analysis. Minneapolis, MN: Pearson.

Porterfield, E. (1978). Black and White mixed marriages: An ethnographic study of Black-White families. Chicago: Nelson-Hall. 
Poston, W. S. C. (1990). The biracial identity development model: A needed addition. Journal of Counseling and Development, 69, 152-155. doi:10.1002/j.1556-6676.1990.tb01477.x

Pyke, K. D. (2010). What is internalized racial oppression and why don't we study it? Acknowledging racism's hidden injuries. Sociological Perspectives, 53(4), 551-572. doi: 10.1525/sop.2010.53.4.551

Rockquemore, K. A., \& Brunsma, D. L. (2002). Socially embedded identities: Theories, typologies, and processes of racial identity among Black/White biracials. Sociological Quarterly, 43(3), 335-356. doi:10.1111/j.1533-8525.2002.tb00052.x

Rockquemore, K. A., Brunsma, D. L., \& Delgado, D. J. (2009). Racing to theory or retheorizing race? Understanding the struggle to build a multiracial identity theory. Journal of Social Issues, 65(1), 13-34. doi:10.1111/j.1540-4560.2008.01585.x

Rockquemore, K.A., \& Laszloffy, T. (2005). Raising biracial children. Lanham, MD: AltaMira. Root, M. P. (1990). Resolving "other" status: Identity development of biracial individuals. Women \& Therapy Special Issue: Diversity and Complexity in Feminist Therapy: I, 9(1-2), 185-205. doi:10.1300/J015v09n01_11

Root, M. P. P. (1996). The multiracial experience: Racial borders as a significant frontier in race relations. In M.P.P. Root (Ed.), The multiracial experience: Racial borders as the new frontier. (pp. xiii- xviii). Thousand Oaks, CA: Sage.

Root, M. P. P. (1997). Multiracial Asians: Models of ethnic identity. Amerasia Journal, 23(1), $29-41$.

Root, M. P. P. (1998). Multiracial Americans: Changing the face of Asian America. In L. C. Lee, \& N. W. S. Zane (Eds.), Handbook of Asian American psychology. (pp. 261-287). Thousand Oaks, CA, US: Sage. 
Biracial Identity Profiles 30

Shih, M., \& Sanchez, D.T. (2005). Perspectives and research on positive and negative implications of having multiple racial identities. Psychological Bulletin, 131(4), 569-591. doi: 10.1037/0033-2909.131.4.569

Statistics Canada (2006). Population groups and sex for the population of Canada, provinces, territories, census metropolitan areas and census agglomerations, 2006 Census - 20\% Sample Data. Catalogue no. 97-562-XCB2006007. Retrieved Sepetmber 28, 2009 from http://www12.statcan.ca/english/census06/data/topics/RetrieveProductTable.cfm

Stevens, J. P. (2002). Applied multivariate statistics for the social sciences (4th ed). Mahwah, NJ: Lawrence Erlbaum.

Rockquemore, K. A., \& Brunsma, D. L. (2002). Socially embedded identities: Theories, typologies, and processes of racial identity among Black/White biracials. Sociological Quarterly, 43(3), 335-356. doi:10.1111/j.1533-8525.2002.tb00052.xSue, D. W., \& Sue, D. (2003). Counseling Asian Americans. Counseling the culturally diverse: Theory and practice (4th ed.) (pp. 327- 342). New York: John Wiley \& Sons.

Tajfel, H., \& Turner, J. (1986). The social identity theory of intergroup behavior. In S. Worchel \& W. Austin (Eds.), Psychology of intergroup relations (pp. 7-24). Chicago: Nelson-Hall. Thornton, M. C. (1996). Hidden agendas, identity theories, and multiracial people. In M. P. P. Root (Ed.), The multiracial experience: Racial borders as the new frontier (pp. 101-120). Thousand Oaks, CA: Sage. Tull, E.S., Sheu, Y., Butler, C., \& Cornelious, K. (2005). Relationships between perceived stress, coping behavior and cortisol secretion in women with high and low levels of internalized racism. Journal of the National Medical Association, 97(2), 206-212. 
Tull, E. S., Wickramasuriya, T., Taylor, J., Smith-Burns, V., Brown, M., Champagnie, G., et al. (1999). Relationship of internalized racism to abdominal obesity and blood pressure in Afro-Caribbean women. Journal of the National Medical Association, 91(8), 447-452.

Uba, L. (1994). Asian Americans: Personality patterns, identity, and mental health. New York: Guilford Press.

Umaña-Taylor, A. J. \& Fine, M. A. (2001). Methodological implications of grouping Latino adolescents into one collective group. Hispanic Journal of Behavioral Sciences, 23(4), 347362. doi: $10.1177 / 0739986301234001$

Umaña-Taylor, A. J.,Yazedjian, A., \& Bámaca-Gómez, M. (2004). Developing the Ethnic Identity Scale using Eriksonian and social identity perspectives. Identity: An International Journal of Theory and Research, 4(1), 9-38. doi:10.1207/S1532706XID0401_2

U.S. Census Bureau (2012). 2012 American Community Survey 1-year estimates. Retrieved from https://www.census.gov/acs/www/ 